\title{
Author Correction: The oxidative inactivation of FeFe hydrogenase reveals the flexibility of the $\mathrm{H}$-cluster
}

Vincent Fourmond, Claudio Greco, Kateryna Sybirna, Carole Baffert, Po-Hung Wang, Pierre Ezanno, Marco Montefiori, Maurizio Bruschi, Isabelle Meynial-Salles, Philippe Soucaille, Jochen Blumberger, Hervé Bottin, Luca De Gioia and

Christophe Léger

Correction to: Nature Chemistry https://doi.org/10.1038/nchem.1892, published online 16 March 2014.

In the version of this Article originally published, in several instances throughout the text, the Chlamydomonas reinhardtii amino acid residue phenylalanine was incorrectly labelled F234; it should have been F290.

\section{Author Correction: Second-generation DNA-templated macrocycle libraries for the discovery of bioactive small molecules}

\author{
Dmitry L. Usanov (D), Alix I. Chan (D), Juan Pablo Maianti and David R. Liu
}

Correction to: Nature Chemistry https://doi.org/10.1038/s41557-018-0033-8, published online 02 April 2018.

In the Supplementary Information file originally published for this Article there were several errors.

In the section 'Typical procedure of preparative split ligation', the sentence " $30-40 \%$ yields" should have read " $30-40 \%$ yields (w/ no extra ATP added)".

In the section 'Large-scale preparative splint ligation', the sentence "Likewise, the additional ATP should be added for higher yields" was missing from the end of the paragraph.

In the section 'Library assembly', the sentence "After $2 \mathrm{~h}$ at $30^{\circ} \mathrm{C}$, each batch was mixed with $6.5 \mathrm{~mL}(25 \% \mathrm{v} / \mathrm{v})$ of $1 \mathrm{M} \mathrm{NaOH} \mathrm{solution}$,

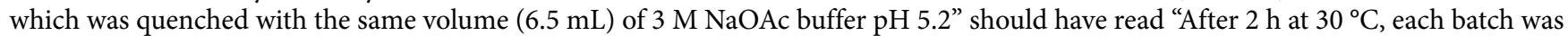
mixed with $6.5 \mathrm{~mL}(25 \% \mathrm{v} / \mathrm{v})$ of $1 \mathrm{M} \mathrm{NaOH}$ solution and left for 30 minutes, followed by quenching with the same volume (6.5 mL) of $3 \mathrm{M} \mathrm{NaOAc}$ buffer $\mathrm{pH}$ 5.2." In the same section, the sentence "The solution in each conical was diluted just with $25 \mathrm{~mL}$ of isopropanol and passed through 14 HiBind DNA Midi columns (Omega Bio-tek)" should have read "The solution in each conical was diluted just with $25 \mathrm{~mL}$ of isopropanol and passed through 14 HiBind DNA Midi columns (7 columns per conical, Omega Bio-tek)”"

In the section 'General procedure for selections and high-throughput sequencing, in the second paragraph, “ $\pm 5 \mathrm{mM}$ DTT” should have read “ $\pm 2 \mathrm{mM}$ TCEP” in two instances.

These errors have now been corrected. 